University of Nebraska - Lincoln

DigitalCommons@University of Nebraska - Lincoln

August 1973

\title{
Comment on "Length and Velocity Formulas in Approximate Oscillator-Strength Calculations"
}

Anthony F. Starace

University of Nebraska-Lincoln, astarace1@unl.edu

Follow this and additional works at: https://digitalcommons.unl.edu/physicsstarace

Part of the Physics Commons

Starace, Anthony F., "Comment on "Length and Velocity Formulas in Approximate Oscillator-Strength Calculations"'" (1973). Anthony F. Starace Publications. 3.

https://digitalcommons.unl.edu/physicsstarace/3

This Article is brought to you for free and open access by the Research Papers in Physics and Astronomy at DigitalCommons@University of Nebraska - Lincoln. It has been accepted for inclusion in Anthony F. Starace Publications by an authorized administrator of DigitalCommons@University of Nebraska - Lincoln. 
with respect to an exact eigenstate of the Hamiltonian, $\mathfrak{H}=T+V$. When the potential is taken to be the GSCP [see Eq. (1)], the virial theorem is reexpressed as

$$
2\langle T\rangle=-\langle V\rangle+\lambda\left\langle\frac{d V}{d \lambda}\right\rangle
$$

In obtaining Eq. (A2), use has been made of the fact that

$$
\frac{d B(\lambda s)}{d s}=-\frac{\lambda}{Z} \frac{d V}{d \lambda}
$$

When the exact wave function for the GSCP is not known, the virial theorem may still be satisfied by utilizing a variational procedure whereby an approximate trial wave function $\psi_{n, l}(Z s)$ and $\lambda$ are subjected to the scale transformation of Eq. (2). ${ }^{11}$ If we use $\psi_{n, l}(Z \eta s)$ as a trial wave function, with $\eta$ as a variational parameter, and let $\rho=\lambda / \eta$, then the approximate expectation values for $T$ and $V$ be- come

$$
\langle T\rangle(\eta)=\eta^{2} \bar{T}
$$

and

$$
\langle V\rangle(\eta)=\eta \bar{r},
$$

where $\bar{T}$ and $\bar{V}$ are defined by Eqs. (4) and (5). The total variational energy then becomes

$$
E_{v}(n, l, \eta, \lambda)=\eta^{2} \bar{T}+\eta \bar{V},
$$

as stated in Eq. (3) and the minimum energy condition of Eq. (9) follows directly. In multiplying the expression in Eq. (9) by $\eta$, we obtain

$$
2 \bar{T} \eta^{2}+\bar{V} \eta-\rho \eta \bar{V}_{\rho}=0 .
$$

Making use of Eqs. (2b), (8), (A4), and (A5), Eq. (A7) becomes

$$
2\langle T\rangle(\eta)=-\langle V\rangle(\eta)+\lambda\left\langle\frac{d V}{d \lambda}\right\rangle(\eta) .
$$

Hence the virial theorem is established, provided that $\eta$ is chosen to satisfy Eq. (A7) .
${ }^{1}$ G. M. Harris, Phys. Rev. 125, 1131 (1962).

${ }^{2}$ H. Margenau and M. Lewis, Rev. Mod. Phys. 31, 569 (1959).

${ }^{3}$ J. B. Krieger, Phys. Rev. 178, 1337 (1969).

${ }^{4} \mathrm{~V}$. Bonch-Bruevich and S. Tyablikov, The Green's Function Method in Statistical Mechanics (Interscience, New York, 1962), p. 161.

${ }^{5}$ G. J. Iafrate and L. B. Mendelsohn, Phys. Rev. 182, 244 (1969).

${ }^{6}$ F. Rogers, H. Grabuske, and D. Harwood, Phys. Rev. A 1, 1577 (1970).

${ }^{7}$ C. R. Smith, Phys. Rev. 134, A1235 (1964).
${ }^{8}$ G. J. Iafrate and L. B. Mendelsohn, Phys. Rev. A 2, 561 (1970).

${ }^{9}$ C. S. Lam and Y. P. Varshni, Phys. Rev. A 6, 1391 (1972).

${ }^{10} \lambda_{c}^{v}$ refers to the value of $\lambda_{c}$ obtained by variational methods.

${ }^{11}$ P. O. Löwdin, J. Mol. Spectrosc. 3, 46 (1959).

${ }^{12}$ The term $\left(E_{I} / \lambda_{c}^{v}\right)$ present in Eqs. (11) and (13) is independent of $\lambda$, as will be shown in a later discussion [see Eq. (34)].

${ }^{13}$ E. Merzbacher, Quantum Mechanics (Wiley, New York, 1961), pp. 373 and 394.

\title{
Comment on "Length and Velocity Formulas in Approximate Oscillator-Strength Calculations"
}

\author{
Anthony F. Starace* \\ Department of Physics, Imperial College, London, S.W.7, England \\ (Received 30 November 1972)
}

\begin{abstract}
A previous paper by Starace showed that the length formula for the electric-dipole matrix element is the only consistent one for calculations that solve for the exact eigenfunctions of an approximate Hamiltonian containing a nonlocal potential. It is emphasized here that disagreement between such a length-formula calculation and experiment indicates inadequacy of the approximate Hamiltonian and not that the velocity formula may be preferable.
\end{abstract}

Starace ${ }^{1}$ has shown that in oscillator-strength calculations employing exact eigenfunctions of an approximate Hamiltonian containing a nonlocal potential, the matrix element for electric-dipole transitions is correctly given by the length formula and not by the velocity (or acceleration) formula. Examples of such approximate Hamiltonians are those of Hartree-Fock and configuration-inter- 
action calculations. This result has been quoted recently by authors who are interested in knowing whether it is the length formula or the velocity formula which gives the best agreement with experiment. ${ }^{2-4}$ More recently Cohen and McEachran ${ }^{5}$ have criticized this result for not predicting which formula gives the best agreement with experiment. To prevent further misunderstandings, it is the purpose of this Comment to discuss briefly what meaning the result of Starace ${ }^{1}$ has, particularly for those who want to know whether it is the length or the velocity formula which gives the best agreement with experiment.

When one approximates the exact Hamiltonian by an approximate Hamiltonian containing a nonlocal potential and also obtains exact eigenfunctions of the approximate Hamiltonian, then it follows from general physical requirements which the approximate Hamiltonian must satisfy ${ }^{1}$ that the matrix element for electric-dipole transitions is given only by the length formula. Any difference between experimental and calculated oscillator strengths is to be interpreted as an indication of some inadequacy of the chosen approximate Hamiltonian. Gambling on the velocity formula to give better agreement with experiment amounts to avoiding this real problem. Indeed, since the velocity formula will in general differ from the length formula in the presence of nonlocal potentials, ${ }^{1}$ it may happen that the velocity formula does sometimes give better agreement with experiment. However, this difference between the length and velocity results is solely a measure of the nonlocality of the approximate potential, as shown, for example, by Chang and $\mathrm{McDowell}^{6}$ for the case of a Hartree-Fock model Hamiltonian.

Two additional points should be noted. Firstly, for the approximations not considered by Ref. 1, most notably variational approximation procedures, the qualitative considerations of Chandrasekhar ${ }^{7}$ and of Crossley ${ }^{8}$ are most valuable and are not in contradiction with Ref. 1. Secondly, it has been pointed out ${ }^{9}$ that in some cases there are numerical problems in computing the electric-dipole matrix element using the length formula. In these cases the alternative expression for the length formula given by Eq. (4) of Ref. 1 may be preferable.

In conclusion, the main result of Starace ${ }^{1}$ is that, once one has started by choosing an approximation procedure of the kind specified above, the usual ambiguity as to which formula should be used to calculate the electric-dipole matrix element no longer exists. In order to be consistent, only the length formula should be used. Any discrepancy between the results of calculations and experiment is to be regarded as due to the physical inadequacy of the chosen approximate Hamiltonian and not of the length formula. That is, for the specific cases of (a) Hartree - Fock calculations or (b) configuration-interaction calculations, any discrepancy between the length calculation and experiment is an indication that (a) Hartree-Fock is not good enough or (b) important configurations have been ignored.
*Present address: Behlen Laboratory of Physics, The University of Nebraska, Lincoln, Neb. 68508.

${ }^{1}$ A. F. Starace, Phys. Rev. A 3, 1242 (1971).

${ }^{2}$ G. W. F. Drake, Phys. Rev. A 5, 614 (1972).

${ }^{3}$ D. J. Kennedy and S. T. Manson, Phys. Rev. A 5, 227 (1972).

${ }^{4}$ Z. Sibincic, Phys. Rev. A 5, 1150 (1972).

${ }^{5}$ M. Cohen and R. P. McEachran, Chem. Phys. Lett. 14, 201
(1972).

${ }^{6}$ E. S. Chang and M. R. C. McDowell, Phys. Rev. 176, 126 (1968).

${ }^{7}$ S. Chandrasekhar, Astrophys. J. 102, 223 (1945).

${ }^{8}$ R. J. S. Crossley, Adv. At. Mol. Phys. 5, 237 (1969).

${ }^{9}$ M. Cohen and R. P. McEachran, Can. J. Phys. 50, 1363 (1972). 